\title{
Pulsars with NenuFAR: Backend and pipelines
}

L. Bondonneau ${ }^{1,14}$, J.-M. Grießmeier ${ }^{1,2}$, G. Theureau ${ }^{1,2,3}$, I. Cognard ${ }^{1,2}$, M. Brionne ${ }^{1}$, V. Kondratiev ${ }^{4,5}$, A. Bilous ${ }^{4}$, J. W. McKee ${ }^{6}$, P. Zarka ${ }^{14,2}$, C. Viou ${ }^{2}$, L. Guillemot ${ }^{1,2}$, S. Chen ${ }^{1}$, R. Main ${ }^{5}$, M. Pilia ${ }^{7}$, A. Possenti ${ }^{7,8}$, M. Serylak ${ }^{9}, 10$, G. Shaifullah ${ }^{4}$, C. Tiburzi ${ }^{4}$, J. P. W. Verbiest ${ }^{11,5}$, Z. Wu ${ }^{11}$, O. Wucknitz ${ }^{5}$, S. Yerin ${ }^{12,13}$, C. Briand ${ }^{14}$, B. Cecconi ${ }^{14,2}$, S. Corbel ${ }^{2,15}$, R. Dallier ${ }^{16,2}$, J. N. Girard ${ }^{15}$, A. Loh ${ }^{14,2}$, L. Martin $^{16,2}$, M. Tagger ${ }^{1}$, and C. Tasse ${ }^{17,2,18}$

${ }^{1}$ LPC2E - Université d'Orléans/CNRS, France

e-mail: louis. bondonneau@obspm. fr

2 Station de Radioastronomie de Nançay, Observatoire de Paris - CNRS/INSU, USR 704 - Univ. Orléans, OSUC, Route de Souesmes, 18330 Nançay, France

${ }^{3}$ Laboratoire Univers et Théories LUTh, Observatoire de Paris, CNRS/INSU, Université Paris Diderot, 5 place Jules Janssen, 92190 Meudon, France

4 ASTRON, the Netherlands Institute for Radio Astronomy, Oude Hoogeveensedijk 4, Dwingeloo 7991 PD, The Netherlands

5 Max-Planck-Institut für Radioastronomie, Auf dem Hügel 69, 53121 Bonn, Germany

${ }^{6}$ Canadian Institute for Theoretical Astrophysics, University of Toronto, 60 St. George Street, Toronto, ON M5S 3H8, Canada

7 INAF-Osservatorio Astronomico di Cagliari, Via della Scienza 5, 09047 Selargius, Italy

8 Universitá di Cagliari, Dipartimento di Fisica, S.P. Monserrato-Sestu Km 0,700, 09042 Monserrato, Italy

9 South African Radio Astronomy Observatory, 2 Fir Street, Black River Park, Observatory 7925, South Africa

10 Department of Physics and Astronomy, University of the Western Cape, Bellville, Cape Town 7535, South Africa

11 Fakultät für Physik, Universität Bielefeld, Postfach 100131, 33501 Bielefeld, Germany

12 Department of Radio Astronomy Equipment and Methods of Observations, Institute of Radio Astronomy of NAS of Ukraine, Kharkiv, Ukraine

13 Department of Astronomy and Space Computer Science, V. N. Karazin Kharkiv National University, Kharkiv, Ukraine

14 LESIA, Observatoire de Paris, CNRS, PSL, SU/UP/UO, 92195 Meudon, France

15 AIM, CEA, CNRS, Université de Paris, Université Paris-Saclay, 91191 Gif-sur-Yvette, France

16 SUBATECH, Institut Mines-Telecom Atlantique, CNRS/IN2P3, Université de Nantes, 44307 Nantes, France

17 GEPI Observatoire de Paris, CNRS, PSL, SU/UP/UO, 92195 Meudon, France

18 Center for Radio Astronomy Techniques and Technologies, Department of Physics and Electronics, Rhodes University, Grahamstown 6140, South Africa

Received 4 September 2020 / Accepted 1 March 2021

\section{ABSTRACT}

\begin{abstract}
Context. NenuFAR (New extension in Nançay upgrading LOFAR) is a new radio telescope developed and built on the site of the Nançay Radio Observatory. It is designed to observe the largely unexplored frequency window from 10 to $85 \mathrm{MHz}$, offering a high sensitivity across its full bandwidth. NenuFAR has started its "early science" operation in July 2019, with 58\% of its final collecting area.

Aims. Pulsars are one of the major phenomena utilized in the scientific exploitation of this frequency range and represent an important challenge in terms of instrumentation. Designing instrumentation at these frequencies is complicated by the need to compensate for the effects of both the interstellar medium and the ionosphere on the observed signal. We have designed a dedicated backend and developed a complete pulsar observation and data analysis pipeline, which we describe in detail in the present paper, together with first science results illustrating the diversity of the pulsar observing modes.

Methods. Our real-time pipeline LUPPI (Low frequency Ultimate Pulsar Processing Instrumentation) is able to cope with a high data rate and provide real-time coherent de-dispersion down to the lowest frequencies reached by NenuFAR (10 MHz). The full backend functionality is described, as the available pulsar observing modes (folded, single-pulse, waveform, and dynamic spectrum).

Results. We also present some of the early science results of NenuFAR on pulsars: the detection of 12 millisecond pulsars (eight of which are detected for the first time below $100 \mathrm{MHz}$ ); a high-frequency resolution mapping of the PSR B1919+21 emission profile and a detailed observation of single-pulse substructures from PSR B0809+74 down to $16 \mathrm{MHz}$; the high rate of giant-pulse emission from the Crab pulsar detected at $68.7 \mathrm{MHz}$ (43 events per minute); and the illustration of the very good timing performance of the instrumentation, which allows us to study dispersion measure variations in great detail.
\end{abstract}

Key words. pulsars: general - methods: observational

\section{Introduction}

Pulsars are rapidly rotating, highly magnetized neutron stars, which emit collimated beams of radiation across the electromagnetic spectrum. Most of the known pulsars have been discovered and studied in the radio band. According to the
Australia Telescope National Facility (ATNF) Pulsar Catalog ${ }^{1}$ (Manchester et al. 2005), about 2800 pulsars have been found to date. Most of them have been discovered in the $L$-band

1 http://www.atnf.csiro.au/people/pulsar/psrcat, V1.63. 
(1-2 GHz) with, for example the High Time Resolution Universe (HTRU) survey (Keith et al. 2010; Cameron et al. 2020), the Parkes Multibeam Pulsar Survey (PMPS; Manchester et al. 2001; Lorimer et al. 2015), and the Pulsar Arecibo L-band Feed Array (PALFA) survey (Lazarus et al. 2015; Parent et al. 2019). A few hundred sources were unveiled by searches around 300-400 MHz (Hessels et al. 2008; Lynch 2013), and recently the low-frequency domain was explored intensively with the LOw Frequency ARray (LOFAR), leading to the discovery of 73 new pulsars in the $119-151 \mathrm{MHz}$ window with the LOFAR Tied-Array All-Sky Survey (LOTAAS; Sanidas et al. 2019).

In the radio band, pulsars are known to have relatively steep spectra, with a power law index in the range of -1 to -3 (Malofeev et al. 2000; Maron et al. 2000; Bilous et al. 2016). Thus, despite the observational challenges caused mostly by the deleterious effects of propagation in the ionized interstellar medium (ISM), low-frequency pulsar surveys are promising. New low-frequency discoveries help sample the low luminosity end of the pulsar population and better explore the properties of the ionized ISM, to which low frequencies are most sensitive. In addition, measuring pulsar flux densities at low radio frequencies helps constrain the so-called low-frequency turnover (Sieber 1973; Malofeev 1993; Bilous et al. 2020), which is often present in pulsar spectra. Finally, the properties of radio emission at low frequencies become very dynamic, providing unique insight into conditions in the pulsar magnetosphere.

Several wideband pulsar studies have recently been performed at frequencies below $100 \mathrm{MHz}$ using pioneering radio telescopes such as LOFAR Low Band Antenna (LBA; Hassall et al. 2012; Pilia et al. 2016; Kondratiev et al. 2016; Bilous et al. 2020; Bondonneau et al. 2020), the Ukrainian T-shaped Radio telescope (UTR-2; Zakharenko et al. 2013) and the Long Wavelength Array (LWA; Stovall et al. 2015). LOFARLBA has a large fractional bandwidth, but highly frequencydependent sensitivity, with antenna response peaking in the $45-75 \mathrm{MHz}$ range. UTR-2 operates at very low frequencies, $10-30 \mathrm{MHz}$, but records a single linear polarization. The LWA has proven to be very powerful, sharing the same antenna radiator design as NenuFAR (Hicks et al. 2012) with a homogeneous frequency coverage across the band, but with only 256 antennas (to be compared with the present 1064 optimized NenuFAR antennas, a number that will eventually increase to 1824).

\section{NenuFAR}

NenuFAR ${ }^{2}$ is a compact phased array and interferometer, formed of hexagonal groups of 19 dual-polarization antennas called mini-arrays (MAs). Its core, of diameter $400 \mathrm{~m}$, today has 56 MAs (1064 antennas). It will include 80 MAs (1520 antennas) by early 2021 and eventually reach 96 MAs (1824 antennas). It is complemented by six MAs located up to $3 \mathrm{~km}$ away, which are not beamformed but used only for imaging (Zarka et al. 2020). The MA spacing and distribution is optimized for UV coverage.

Similarly to LOFAR High Band Antenna (HBA; see van Haarlem et al. 2013), NenuFAR is designed to deal with the received radio signal on three different levels. While single antennas see the entire sky above them, analogically phased MAs of 19 antennas have a field of view of 8 to 69 degrees depending on the frequency $(85-10 \mathrm{MHz})$. This can be computed from the full width at half maximum (FWHM) $\frac{\lambda}{D}$, where $\lambda$ is the wavelength and $D$ is the MA diameter, $\approx 25 \mathrm{~m}$ (see Table 1).

2 https://nenufar.obs-nancay.fr/en/astronomer
Table 1. Summary of NenuFAR core elements.

\begin{tabular}{lccc}
\hline \hline & Size & $\begin{array}{c}\text { FoV } \\
(10-85 \mathrm{MHz})\end{array}$ & Sensitivity \\
\hline Single antenna & $\sim 2 \mathrm{~m}$ & $\sim$ full sky & $5500 \mathrm{mJy}$ \\
\hline $\begin{array}{l}\text { Mini-Array (MA) } \\
\text { Analog beam }\end{array}$ & $25 \mathrm{~m}$ & $69-8^{\circ}$ & $290 \mathrm{mJy}$ \\
\hline $\begin{array}{l}\text { NenuFAR } \\
\text { Digital beam }\end{array}$ & $400 \mathrm{~m}$ & $4.3-0.5^{\circ}$ & $\begin{array}{l}5 \mathrm{mJy}(56 \mathrm{MA}) \\
3 \mathrm{mJy}(96 \mathrm{MA})\end{array}$ \\
\hline
\end{tabular}

Notes. Size: the dimension or longest baseline of the element. FoV: the 1D field of view range from 10 to $85 \mathrm{MHz}$. Sensitivity: minimal detectable mean flux density as described in Sect. 2.1 assuming $4 \mathrm{~h}$ of integration time and $75 \mathrm{MHz}$ of bandwidth.

The full NenuFAR core, with its $400 \mathrm{~m}$ diameter, allows the synthesization of digital beams that are 0.5 to 4.3 degrees wide. To ensure smooth source tracking, analog beams are re-pointed every $6 \mathrm{~min}$ through a pointing grid of $128 \mathrm{E}-\mathrm{W} \times 128 \mathrm{~N}-\mathrm{S}$ steps, while digital beams are re-pointed every $10 \mathrm{~s}$ by the beamformer.

The geocentric coordinates of the phase center are the same as for LOFAR international station FR606, that is in the ETRS89 (or WGS84 Cartesian) reference frame: $(x, y, z)=$ (4324017.054, 165545.160, 4670271.072 m). The time reference comes from a rubidium clock source in Nançay. The main NenuFAR backend digitizes MA signals at a sampling frequency of $200 \mathrm{MHz}$ and channelizes them in 1024 bands of $195.3125 \mathrm{kHz}$ (hereafter $195 \mathrm{kHz}$, for simplicity). Then it beamforms the channelized MA signals, that is, it performs their coherent summation with adequate phase shifts in order to form beamlets of $195 \mathrm{kHz}$ bandwidth, each at a center frequency $f_{\mathrm{c}}$ and pointed to a direction $(\theta, \phi)$ in the sky, within the analog beam. Beamlet signals are streams of 195k-complex pairs per second, with time resolution $5.12 \mu \mathrm{s}$, in 8-bits or 16 bits (the pulsar backend currently uses the 8 bit mode only), allowing for further processing and computation of the four Stokes parameters of the incident waves.

The NenuFAR beamformer produces 768 such beamlets, with any selection of $\left(f_{\mathrm{c}}, \theta, \phi\right)$ per beamlet, corresponding to a total bandwidth of $150 \mathrm{MHz}$. It is thus possible to form two digital beams in the sky (each covering the full band of the telescope, $75 \mathrm{MHz}$ ), four beams of $37.5 \mathrm{MHz}$ bandwidth each, or any other combination. By default, all digital beams use all MAs. As the 19 antennas composing an MA are phased analogically, the ana$\log$ beam of an MA can only be pointed in one direction only at a time. However it is possible to form several analog phases pointed to different directions by dividing NenuFAR into multiple sub-arrays (each corresponding to a different set of MAs). This allows us to observe in fly's eye mode with up to four totally independent directions on the sky, corresponding to four analog beams.

For all cases, the full polarization information of the signal is recorded in the form of four Stokes parameters. It is possible to observe up to $8 \mathrm{~h}$ in a row, and there is no constraint on chaining several successive observations. More details on NenuFAR, its beamformer mode, flux and polarization calibration will be described in the full instrument paper (Zarka et al., in prep.).

In 2020 , NenuFAR gradually moved to a $90 \%$ duty cycle at night ( $18 \mathrm{~h}-6 \mathrm{~h}$, local time) and $40 \%$ during the day. The remaining time is used for calibration observations, tests, maintenance, deploying new antennas, and re-observations in the case of errors or radio frequency interference (RFI) excess. 


\subsection{Mean flux density limit}

The detection limit of NenuFAR for pulsars can be estimated using the radiometer equation (Lorimer \& Kramer 2004) with the same set of parameters as in Bondonneau et al. (2020) for the LOFAR single station FR606, but using the collecting area and the instrument temperature of NenuFAR for 56 MAs as well as the ultimate 96 MAs version. The result is shown in Fig. 1 which compares the minimal flux density detectable by FR606 (red curve) with the corresponding limit of NenuFAR for 56 MAs (yellow curve) and 96 MAs (blue curve). In this figure the minimum detectable flux density is calculated for a pointing outside the Galactic plane $\left(g l=0^{\circ}, g b=90^{\circ}, T_{\text {sky }}=2350 \mathrm{~K}\right)$, assuming $4 \mathrm{~h}$ of integration time, $75 \mathrm{MHz}$ of bandwidth, a $\mathrm{S} / \mathrm{N}$ of 5 , and a pulse duty cycle of $7 \%$. The pulsars displayed were detected by the FR606 station with duty cycles greater than $7 \%$ (Bondonneau et al. 2020) and have been re-detected by NenuFAR. There is a large jump in sensitivity between the instruments, which is mainly due to a factor of 11 in the collecting area. This jump will allow for a large number of new detections with the NenuFAR targeted survey.

\subsection{RFI environment at NenuFAR site}

Like all radio telescopes NenuFAR is subject to RFI. The postprocessing pipeline for the folded mode (Sect. 3.4.1) is equipped with an automatic RFI cleaner (Sect. 3.5.2). Figure 2 shows RFI statistics across the NenuFAR band. Our observations show a remarkably clean sky between 38 and $72 \mathrm{MHz}$. A few frequencies are permanently occupied by narrowband RFI (e.g. 35.5 MHz, 72.5 MHz, and 78 MHz). Above 87.5 MHz, the band is saturated by frequency modulation broadcast. Below $30 \mathrm{MHz}$, Fig. 2 shows broadband interference $(10-25 \mathrm{MHz})$ that is much brighter and extends to higher frequencies in the daytime than during the nighttime. The usually observed band $(10-85 \mathrm{MHz})$ remains fairly clean with $\sim 82 \%$ of the band usable during daytime and $\sim 87 \%$ at nighttime. During the daytime, RFI heavily affects the observations of pulsars below $20 \mathrm{MHz}$; at nighttime, observations down to $10 \mathrm{MHz}$ remain possible.

\section{Pulsar instrumentation}

\subsection{De-dispersion}

The main effect of the ionized ISM is the dispersion of the radio waves, where the dispersive delay is proportional to the line-ofsight integrated electron content, the so-called dispersion measure (DM). The dispersive delay, also being also proportional to $v^{-2}$ (where $v$ is the observing frequency) has a strong impact on low-frequency radio observations and requires dedicated instrumentation to process the signal and detect pulsed emission.

There are two ways to correct for dispersive delays (see e.g. Lorimer \& Kramer 2004; Hankins \& Rickett 1975). Incoherent de-dispersion corrects for inter-channel delays only and requires a large number of narrow channels to minimize intra-channel smearing. It is generally applied off-line, once the signal has been recorded. Coherent de-dispersion is designed to correct for intra-channel delay, managing the waveform signal by changing its phase in the Fourier domain. This process makes it possible to have wide channels while keeping a high time resolution. This method does not introduce dispersion smearing into the data in contrast with incoherent de-dispersion. It is clear that coherent de-dispersion is necessary to preserve the integrity of the pulsar pulse profile, especially at low frequencies. To correct for the

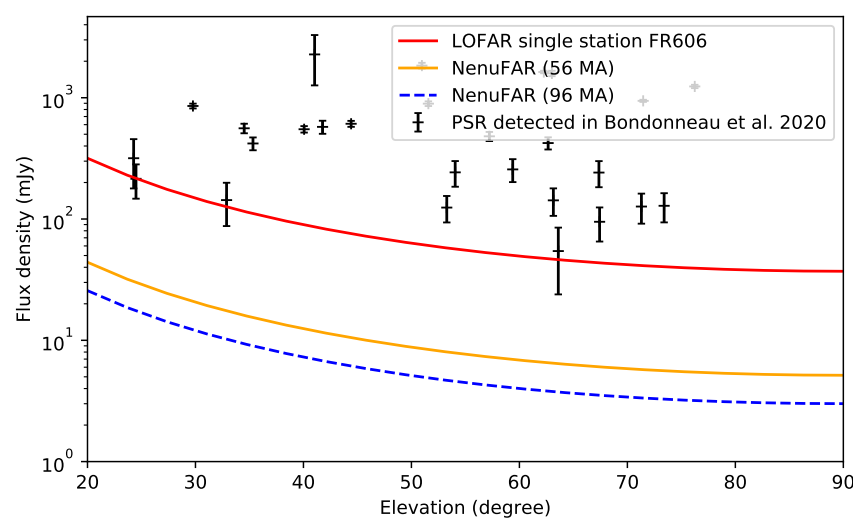

Fig. 1. Minimum detectable flux density as a function of source elevation (see text for details). The measured mean flux density of the pulsars with duty cycles greater than $>7 \%$ detected with FR606 (Bondonneau et al. 2020) are indicated in black.

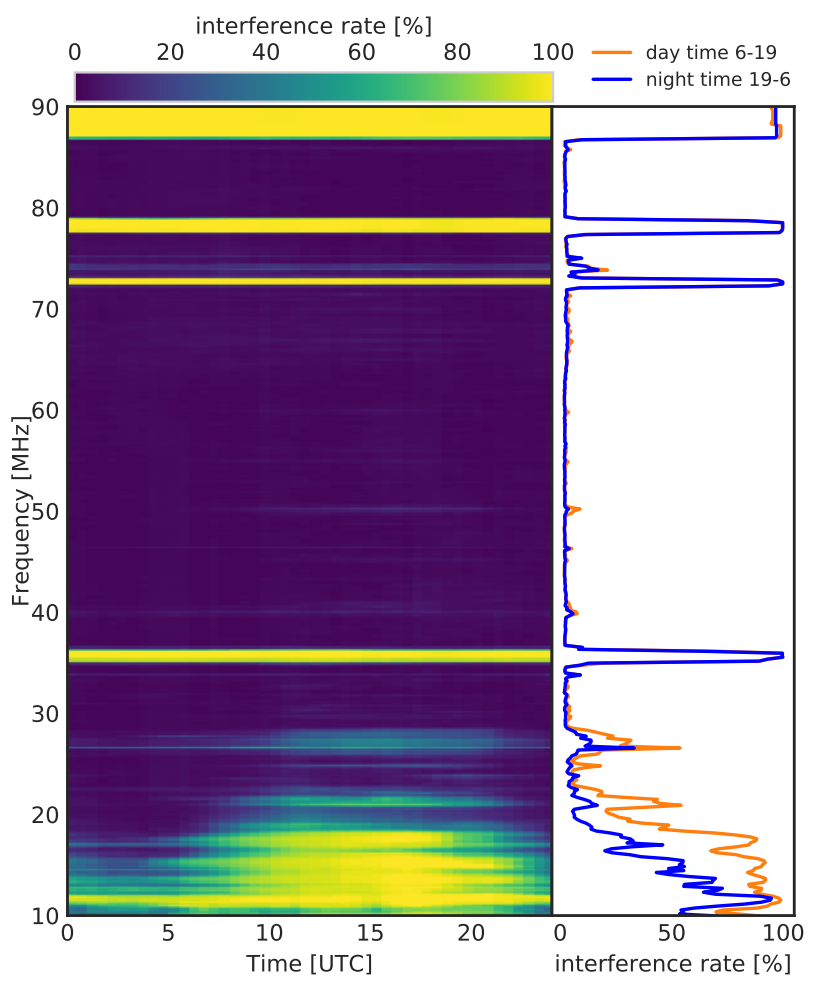

Fig. 2. Radio frequency interference statistics for NenuFAR computed from over $2500 \mathrm{~h}$ of observing time (2018-2020). Left panel: dynamic spectrum of the RFI rate (percentage of data removed during RFI cleaning) as a function of the time of day (in UTC) and frequency. Right panel: spectrum of the RFI rate during daytime (orange, 6-19UT) and nighttime (blue, 19-6 UT).

intra-channel dispersion at low frequencies $(10-85 \mathrm{MHz})$ this method requires long Fourier transforms and a coherent dispersion code dimensioned for the corresponding buffer.

The lowest frequency channel that can be coherently de-dispersed is directly related to the block size of the instrumentation $\left(\approx 10\right.$ s without overlap $\left.{ }^{3}\right)$ and the intra-channel dispersive delay corresponding to the DM of the observed pulsar, that is,

3 The overlap: a duplication of the ends of the data series to take into account the differences in dispersive delays between the top and bottom of the lowest frequency channel during the FFT. 
$12 \mathrm{MHz}$ for $\mathrm{DM} \sim 10 \mathrm{pc} \mathrm{cm}^{-3}, 25 \mathrm{MHz}$ for $\mathrm{DM} \sim 100 \mathrm{pc} \mathrm{cm}^{-3}$, and $53 \mathrm{MHz}$ for $\mathrm{DM} \sim 1000 \mathrm{pc} \mathrm{cm}^{-3}$ (see Sect. 3.3).

\subsection{Faraday de-rotation}

Faraday rotation is a frequency-dependent phase shift between right and left circular polarization. It is a consequence of the parallel component of the magnetic field and the electronic density along the line of sight, the so-called rotation measure (RM). This effect results in a rotation of the polarization plane by an angle $\beta$, where $\beta$ is proportional to $v^{-2}$. The Faraday rotation has a strong impact on low-frequency radio observations and requires dedicated instrumentation to process the signal and detect linear polarization. As for dispersion, there are two ways to correct for Faraday rotation. Incoherent Faraday de-rotation corrects only for the inter-channel rotation of the polarization plane and requires a large number of narrow channels to minimize intra-channel depolarization. Coherent Faraday de-rotation is designed to correct for intra-channels rotation of the polarization plane, managing the waveform signal by applying a Jones matrix per complex voltage in the Fourier domain. This technique is already implemented in the NenuFAR backend and will be described in Bondonneau et al. (in prep.).

\subsection{The UnDySPuTeD LUPPI}

The new real-time code Low frequency Ultimate Pulsar Processing Instrumentation (LUPPI; Bondonneau et al. 2018) operating on NenuFAR was adapted from the software used for Nançay Ultimate Pulsar Processing Instrument (NUPPI, Desvignes 2014). NUPPI is the graphical processing unit (GPU) cluster for the Nançay Radio Telescope (NRT) pulsars observations since August 2011. NUPPI is designed to handle dispersive delays in $4 \mathrm{MHz}$ channels in $L$ - or $S$-bands for a total instantaneous bandwidth of $512 \mathrm{MHz}$. The NUPPI software was derived from Green Bank Ultimate Pulsar Processing Instrument (GUPPI; Demorest 2014; DuPlain et al. 2008).

LUPPI runs on two composite machines, which together are named UnDySPuTeD (Unified Dynamic Spectrum Pulsar and Time Domain receiver). Each hosts two Intel Xeon E5-2620 central processing units (CPUs) and two Nvidia GeForce GTX 1080 GPUs.

By using the two UnDySPuTeD machines together, LUPPI has at its disposal a total of $32 \mathrm{CPU}$ cores, $512 \mathrm{~GB}$ of errorcorrecting code RAM memory, and $32 \mathrm{~GB}$ of GPU memory. Because of the observing frequencies below $100 \mathrm{MHz}$, LUPPI has to deal with additional difficulties compared to GUPPI and NUPPI.

The huge intra-channel dispersion imposes extremely long Fourier transforms (typically more than $20 \mathrm{~s}$ with the overlap, i.e., 200 times longer than for similar computations at $1.4 \mathrm{GHz}$ ) and leads to memory management difficulties (both RAM and GPU) in the NenuFAR framework. These difficulties have been mitigated by modifying the size of the buffers as well as the variables that contain this information in the source code. These modifications enable the correction of intra-channel dispersive delays of up to $10.737 \mathrm{~s}$.

LUPPI captures the 768 beamlets produced by the NenuFAR beamformer (see Sect. 2) as a series of network packets. The two complex-voltage values (one per polarization) at each time step are used to compute the four Stokes parameters of the signal.

The operational scheme of LUPPI is shown in Fig. 3. The pipeline is divided into four real-time elements and a postprocessing analysis.

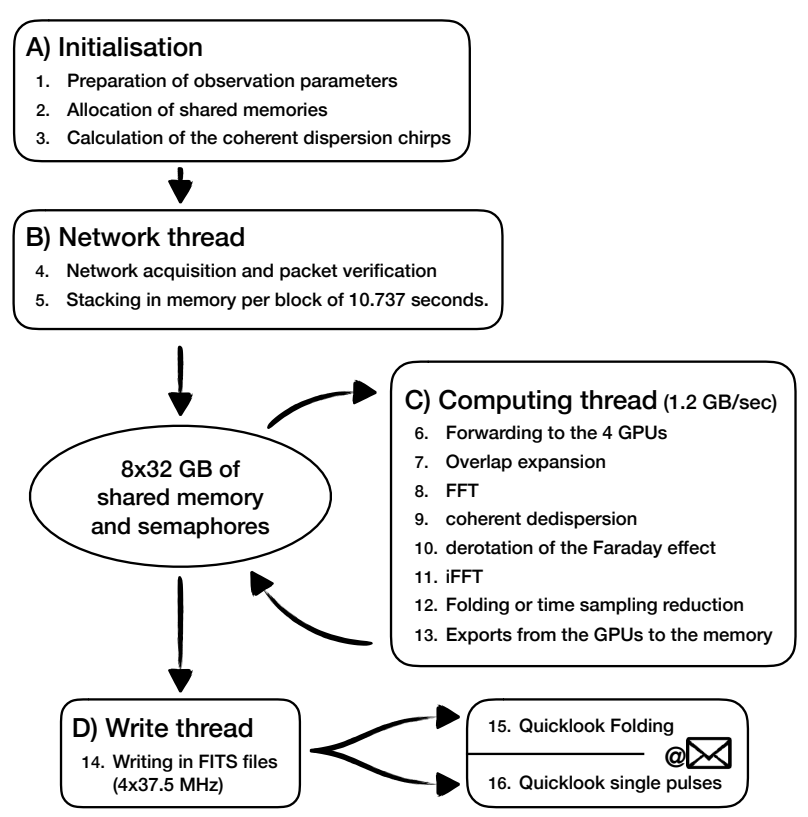

Fig. 3. Operational scheme of the LUPPI pipeline, including its main elements: initialization (A) of the observation parameters, the data processing cycle organized in threads around a shared memory and communicating through semaphores, and post-processing of the observation with production of "quicklook" plots. The network thread (B) catches the packets sent by the beamformer, the computing thread (C) processes the data in the Fourier domain on GPUs, and the write thread (D) exports data products to disks.

The initialization phase (A) prepares the parameters of the observation (DM, channels, source name, etc.) and allocates a total of $128 \mathrm{~GB}^{4}$ of shared memory. We used shared memory to implement ring buffers ${ }^{5}$ in order to allow threads to exchange data and process in parallel. It is during this phase that the coherent dispersion chirps ${ }^{6}$ are calculated.

The network thread (B) catches user datagram protocol (UDP) packets sent by the beamformer, reads the packet index to look for any missing packets and takes it into consideration to preserve the linearity of the time series. The packets are then stored in the shared memory, and a semaphore is used to unleash the computation thread.

The computation thread (C) is dedicated to feeding GPUs with data and retrieving the results. At this stage, the data blocks are sent to four GPUs (one processing unit per band of 37.5 MHz).

First, a fixed overlap is added to absorb the intra-channel dispersive delay. For given GPU hardware, the value of the overlap is a result of a compromise between computing efficiency (which is better when the overlap is small) and the possibility of reaching low frequencies (for a given DM value, a larger overlap allows data to be processed at a lower frequency). With

4 Buffer size: $4(4 \times 37.5 \mathrm{MHz}) \times 2$ (input and output buffer $) \times 4$ (number of blocks per buffer) $\times 2$ (dual linear polarization) $\times 2$ ( 2 byte complex) $\times 2^{22}$ (number of points per FFT) $\times 192$ (number of channels) $\simeq 8 \times 12.88 \mathrm{~GB}$. The buffer size is rounded up to $8 \times 32 \mathrm{~GB}$ to be compatible with a future 16-bit mode.

5 The ring buffer: a structure for storing data in memory where the last block is topologically adjacent to the first one, giving it its ring characteristic.

6 The chirp: transfer function for the phase changes of the coherent dispersion in the Fourier domain. As the chirp is frequency-dependent, there are as many chirps as there are channels per single DM. 
our GPUs being limited to 8 GB of memory, we chose an overlap of $50 \%$ of the total block size (i.e. 10.737 s). With this, can de-disperse frequency channels of frequency $>12 \mathrm{MHz}$ for a DM of $\sim 10 \mathrm{pc} \mathrm{cm}^{-3},>25 \mathrm{MHz}$ for a DM of $\sim 100 \mathrm{pc} \mathrm{cm}^{-3}$, and $>53 \mathrm{MHz}$ for a DM of $\sim 1000 \mathrm{pc} \mathrm{cm}^{-3}$.

Then, the complex voltages are transformed to the Fourier domain using the NVIDIA CUDA Fast Fourier Transform library $^{7}$ in order to multiply the signal by the transfer function corresponding to the coherent dispersion chirp. One of the unique features of this instrumentation is the availability of a Faraday coherent de-rotation (see Sect. 3.2) which allows the rotation of the linear polarization to be corrected for within each channel (Bondonneau et al., in prep.).

Furthermore, the data are transformed back to the time domain with an inverse Fast Fourier Transform (iFFT) and folded at the period of the observed pulsar or integrated in time depending on the observing mode. Finally, the result is pushed into the output buffer and a semaphore is activated to launch the write thread (D).

The write thread writes out the data product from the output buffer into a redundant array of independent disk 0 (RAID 0) storage unit using data striping.

The last step is the post-processing to verify that the observation output products are present on the server and to create diagnostic plots ("quicklooks", see Sect. 3.5.4). The quicklook plots are sent by email to the observer within minutes. If data files are missing on the server, a warning email is sent to the observer. The source code of LUPPI is available on Github ${ }^{8}$.

\subsection{Currently available observing modes}

For the observation of pulsars, NenuFAR currently offers the modes described below.

\subsubsection{Folded mode}

Time series are coherently de-dispersed in every $195 \mathrm{kHz}$ channel and folded at the apparent period of a pulsar using an upto-date ephemeris. The default sub-integration time is $10.737 \mathrm{~s}$. Data are stored in a standard flexible image transport system (FITS) based format for pulsar data files (i.e., PSRFITS ${ }^{9}$ Hotan et al. 2004) and a quicklook plotting program displays the main features of the observation after the removal of RFI. This is the main observing mode. It is used to characterize the pulse profile and polarization as a function of frequency, analyze the spectral energy distribution, and study long-term variations, for example those related to DM fluctuations or intrinsic behavior in the pulsar magnetosphere.

\subsubsection{Single-pulse mode}

In this mode a time series is de-dispersed and integrated in time by a chosen factor (128 by default) to enable the analysis of pulse-to-pulse variations. Data are stored in PSRFITS format and a quicklook program displays the main features of the observation after RFI removal (using the rfifind software from PRESTO ${ }^{10}$; Ransom 2001). This mode is used to observe

\footnotetext{
cuFFT https://docs.nvidia.com/cuda/cufft

8 https://github.com/louisbondonneau/LUPPI

9 https://www.atnf.csiro.au/research/pulsar/psrfits_ definition/Psrfits.html

${ }^{10}$ PRESTO: a toolbox developed to search for single-pulses and periodic pulsations in pulsar observations (see Ransom 2001).
}

bright pulsars that show interesting features in their emission beam, such as drifting sub-pulses, mode changing, or anomalously intense pulses. It will also be used to search for pulsed emission when the DM is higher than $100 \mathrm{pc} \mathrm{cm}^{-3}$ and the scattering is important. Moreover, single-pulse data can easily be converted to folded data.

\subsubsection{Waveform mode}

This mode consists in writing the raw complex-voltage data to disk, bypassing the computing thread in Fig. 3. It keeps the full time, frequency, and polarization information from the original raw signal (thus a very large data rate). This mode can be used to perform non-standard analyses, such as scattered giant pulse (GP) searches, a census of globular clusters' pulsar populations, or interstellar and inter-binary scintillation studies. Additionally, waveform data can be converted to folded or single-pulse data by coherent de-dispersion and integration.

\subsubsection{Dynamic spectrum mode}

The $195 \mathrm{kHz}$ wide beamlets sent by the beamformer are GPUprocessed to provide finer frequency resolution and time integration. The frequency resolution can be chosen between 0.10 and $12.20 \mathrm{kHz}$, corresponding to fast Fourier transform (FFT) lengths from 2048 to 16 samples. The time resolution (corresponding to a number of integrated FFTs restricted to be a power of two, and being at least four) can be chosen between 0.30 and $84.0 \mathrm{~ms}$ (depending on the frequency resolution). A few of the available apodization functions (windowing) can be applied (such as Hann, Hamming, or others), the default being Hamming. The resulting dynamic spectra are recorded in a very simple binary format. This dynamic spectrum mode is used for solar or Jupiter observations, for exoplanet searches, and in the context of a pulsar blind survey or any radio transient search. This mode can be used to observe pulsars without de-dispersion, for instance the bright pulsar B1919+21 (Fig. 4). In addition, it supports very fine channels (e.g., $1.5 \mathrm{kHz}$ ), enabling searches over wider DM ranges without coherent de-dispersion (see a detection example in Fig. 5).

\subsubsection{Compressed UDP waveform mode}

For a small number of projects, we used a second waveform mode, in which UDP packets are directly dumped to disk when they arrive on the network socket. In this mode, we use real-time lossless compression with $\mathrm{zstd}^{11}$, which allows us to reduce the data volume by a factor of $\sim 2-3$. Files written in this mode do not contain any header information. This mode is used, for example, for low-frequency very Long baseline interferometry (VLBI) with international LOFAR stations on baselines of $\sim 1000 \mathrm{~km}$.

\subsection{The post-processing pipeline}

In order to analyze observations, we developed two postprocessing pipelines for folded and single-pulse observations. The role of these pipelines is to ensure that the transfer between the real-time processing machine and the data storage is successful and to redirect the data into the different branches of processing according to their mode of observation: waveforms, folded, or single-pulses.

\footnotetext{
11 https://github.com/facebook/zstd
} 

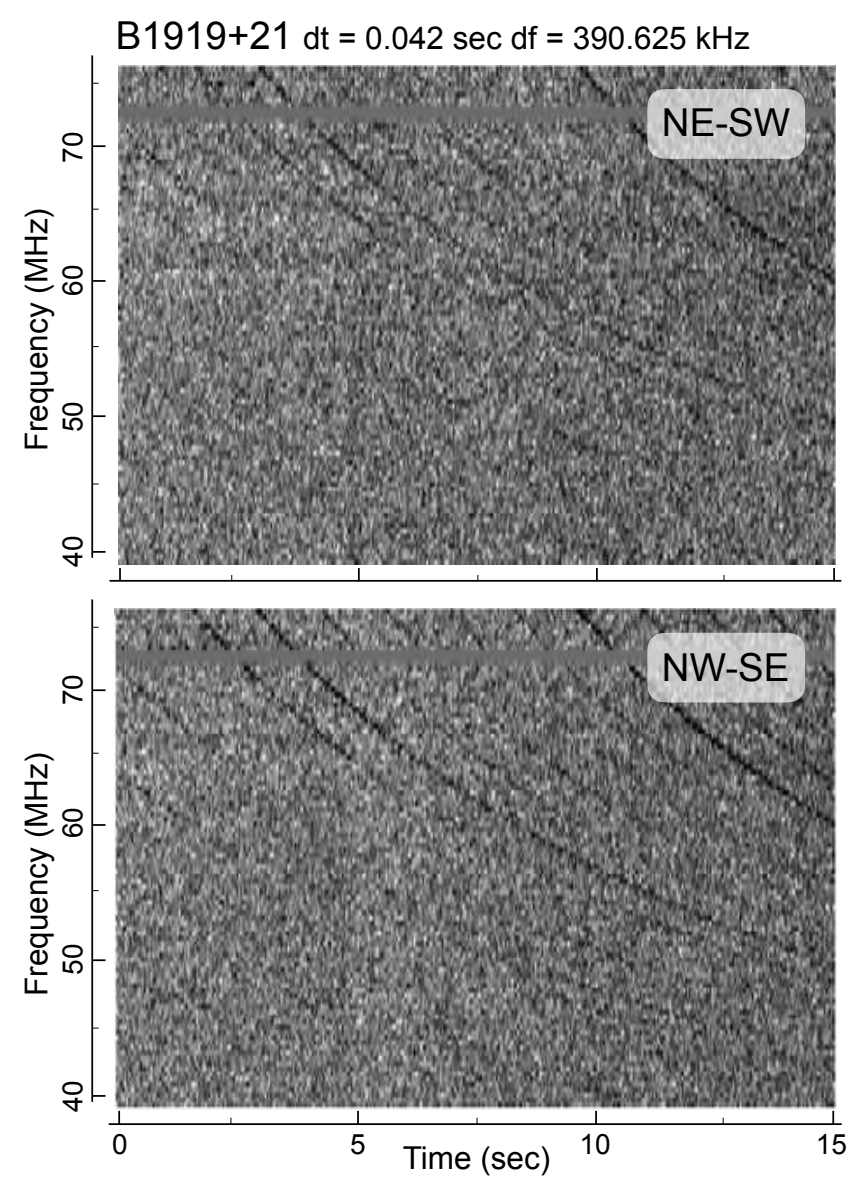

Fig. 4. Dynamic spectrum of a $15 \mathrm{~min}$ observation pointing on PSR B1919+21. Top and bottom panels: provide the linear feed polarization from the NE-SW and NW-SE dipoles corresponding to XX and YY, respectively. These dynamic spectra were recorded with time and frequency resolutions of $0.01 \mathrm{~s}$ and $1.5 \mathrm{kHz}$, and then integrated to $0.04 \mathrm{~s}$ and $390 \mathrm{kHz}$ for this display.

\subsubsection{Waveform mode}

The waveform mode corresponding to the raw data requires large processing and storage resources (540 GB per hour for a bandwidth of $37.5 \mathrm{MHz}$ ). Consequently, it is not handled by the quicklook pipeline even if the transfer remains automatic.

\subsubsection{Folded mode}

The post-processing pipeline for the folded observations is based on Nenuplot.py ${ }^{12}$. Based on the PSRCHIVE ${ }^{13}$ library. This program is fed with the folded FITS files to perform the RFI mitigation and get out a quicklook in PNG format, as described in the next section. Finally, the quicklook is automatically sent by email to the observer. It is described in more detail in Sect. 3.5.4.

\footnotetext{
${ }^{12}$ Nenuplot.py: a completely automatized pipeline for folded observations. Based on the PSRCHIVE library (Hotan et al. 2004), this code is able to rapidly mitigate RFIs in the observation (using a modified version of the CoastGuard software; Lazarus et al. 2016, https://github.com/plazar/coast_guard) and create a quicklook. The source code is available on Github (https://github.com/ louisbondonneau/NenuPlot).

13 PSRCHIVE : a library for the analysis of pulsar astronomical data. It implements an extensive range of algorithms for use in pulsar timing, scintillation studies, polarimetric calibration, single-pulse work, RFI mitigation, etc. The software is described in Hotan et al. (2004).
}

\subsubsection{Single-pulse mode}

Single-pulse observations are converted from 32 bits to 8-bits and normalized in time and frequency domains with a FITE file converter ${ }^{14}$. Furthermore, the block size of a PSRFITS file is reduced with RescaleTime. py ${ }^{15}$ in order to allow more accurate RFI mitigation with rfifind. Then, the PSRFITS file is analyzed with PRESTO tools using a fine DM grid adapted to low frequencies to search for intense pulses in time and DM. PRESTO produces a quicklook analog to that shown in Fig. 6, which is sent by email to the observer. Moreover, we use dspsr ${ }^{16}$ to transform single-pulse 8-bit PSRFITS files into folded and dedispersed PSRFITS file, which allows us to use Nenuplot.py to produce a quicklook (see in Sect. 3.5.4) and send it to the observer with the PRESTO quicklook.

\subsubsection{The quicklook}

The creation of a quicklook is a crucial part of the observation because it makes it possible to monitor the operation of the pipeline as well as the quality of the observation with respect to, for example, RFI. Its aims are to allow a quick validation of an observation and to reduce the response time in the case of a problem.

This python software is based on the PSRCHIVE library, and it is designed to be a complete pipeline compatible with all radio telescopes that generate folded PSRFITS files. A quicklook of an observation of PSR B0329+54 is shown in Fig. 7. The quicklook plot presents the observation data in all its dimensions - time, phase, frequency, and polarization - in various panels. Panel 1 shows the pulse profile with the total intensity (in black), the linear polarization (in red), and the circular polarization (in blue). The profile allows us to determine if the observed source is detected or not. Panel 2 shows the metadata of the observation including the source name, DM, RM, pulsar spin period, observation duration, bandwidth, signal-tonoise ratio $(\mathrm{S} / \mathrm{N})$, percentage of $\mathrm{RFI}$, elevation of the source, etc. Panel 3 shows the temporal variation of the frequency-integrated profile. This plot is useful for highlighting changes in the pulsar period with respect to the reference ephemeris used for folding the observation or a time variation of the pulse profile. Panel 4 is the time-integrated bandpass (i.e., the spectral response of the telescope) for the polarization parameters XX and YY (in blue and red, respectively). Channels flagged because of the presence of RFI are highlighted in gray in this panel. Panel 5 shows the time-integrated profile as a function of frequency for Stokes $I$ (total intensity). This plot is important because, at low frequency, the observations are particularly sensitive to changes in DM. Panels 6-8 show the frequency-resolved profiles for Stokes parameters $Q, U$, and $V$. The color corresponds to the sign of the parameter (red for positive values and blue for negative). These plots are helpful for characterizing the RM used to correct for the Faraday rotation between channels. In the example of

14 ChangeFormat_rescale.py: This code is designed to convert 32-bit PSRFITS data into 8-bit data while maintaining the signal fidelity. The signal is transformed to 8-bit by decomposing it in data, scale, and offset $($ signal $=($ data $\times$ scale $)+$ offset $)$, which allows us to maintain the dynamic range of the signal. The source code is available on Github (https://github.com/louisbondonneau/psrfits_search).

15 RescaleTime.py: The objective of this code is to reduce the block size in the PSRFITS file. The source code is available on Github (https://github.com/louisbondonneau/psrfits_search).

16 DSPSR (van Straten \& Bailes 2011) is an open Source library. It is available on Github (https://github. com/demorest/dspsr). 


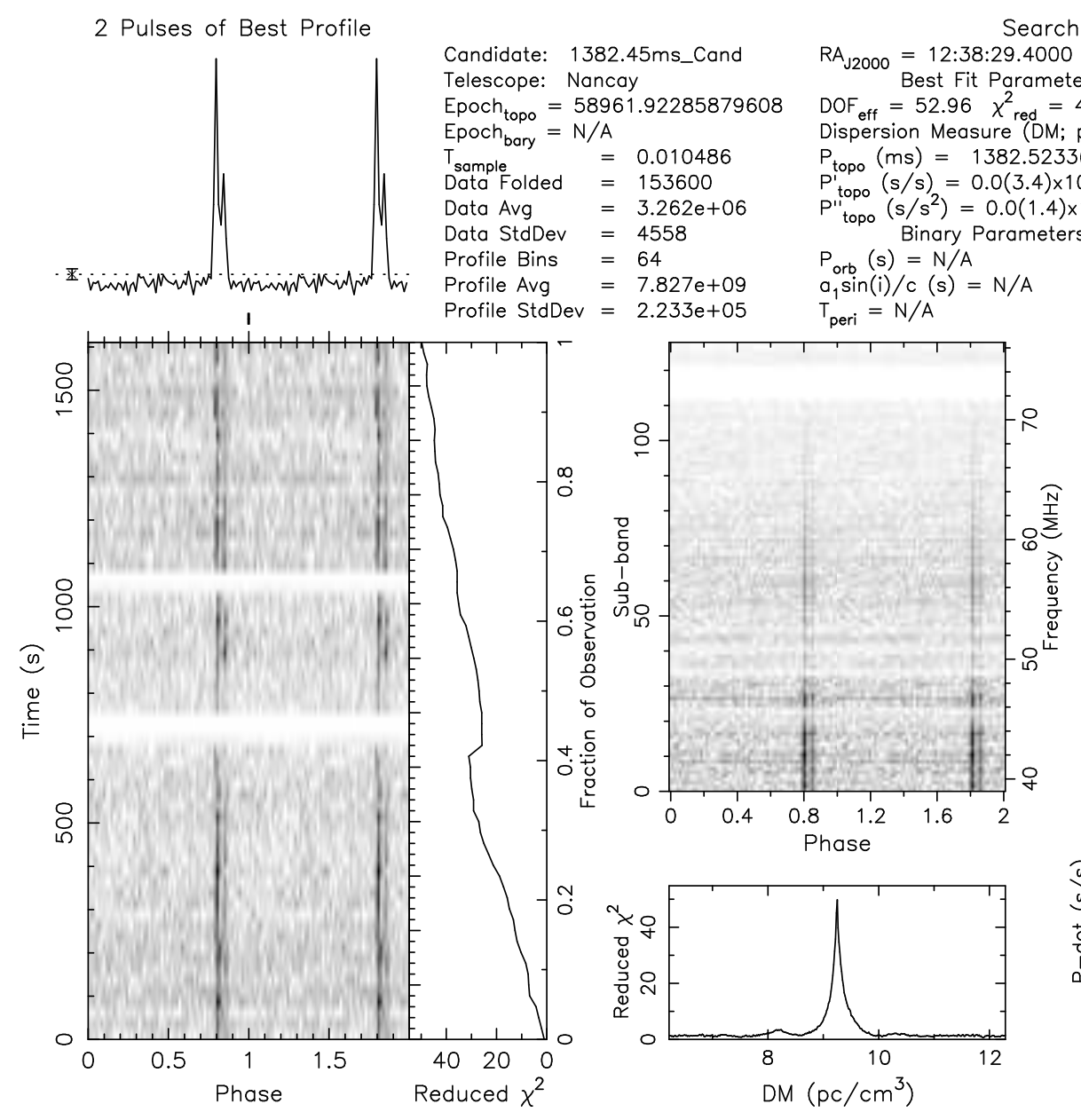

Search Information

$$
\begin{aligned}
& D_{\mathrm{J} 2000}=25: 50: 38.1000 \\
& \mathrm{DOF}_{\text {eff }}=52.96 \chi_{\text {red }}^{2}=49.802 \mathrm{P}(\text { Noise }) \sim 0 \quad(53.2 \sigma) \\
& \text { Dispersion Measure (DM; } \left.\mathrm{pc} / \mathrm{cm}^{3}\right)=9.252 \\
& P_{\text {topo }}(\mathrm{ms})=1382.5233(70) \quad P_{\text {bary }}(\mathrm{ms})=\mathrm{N} / \mathrm{A} \\
& \mathrm{P}_{\text {topo }}^{\text {topo }}(\mathrm{s} / \mathrm{s})=0.0(3.4) \times 10^{-8} \quad \mathrm{P}_{\text {bary }}^{\prime}(\mathrm{s} / \mathrm{s})=\mathrm{N} / \mathrm{A}
\end{aligned}
$$

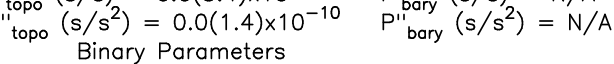

$$
\begin{aligned}
& e=N / A \\
& a_{1} \sin (i) / c(s)=N / A \quad \omega(\mathrm{rad})=N / A
\end{aligned}
$$
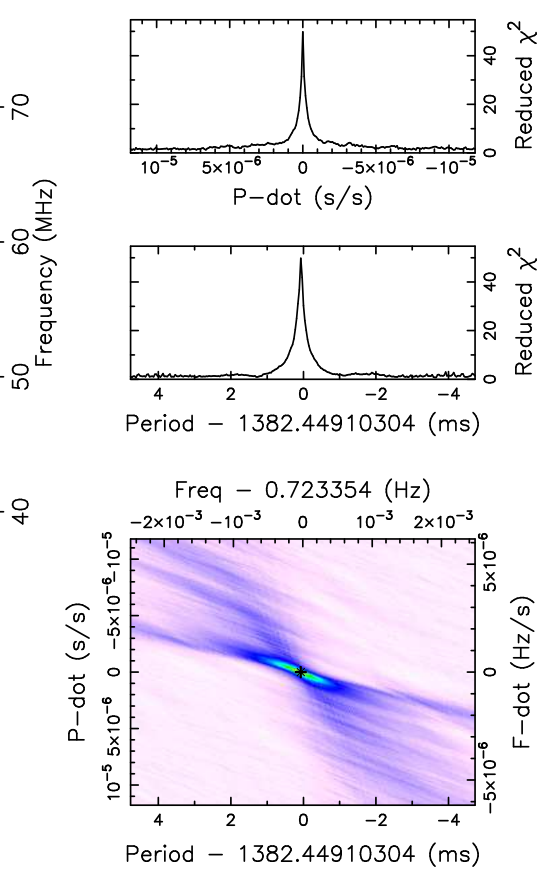

Fig. 5. Detection plot of PSR B1237+25, the first candidate provided from the PREST0 analysis of a 30 min observation. The blind search was conducted over DMs between 1.0 and 60.0 with steps of $0.01 \mathrm{pc} \mathrm{cm}^{-3}$. Top-left panel: detection profile over two periods for the best DM and pulsar spin period, P0. Bottom-left panel: time vs. phase diagram for the best DM and P0. The right part of the bottom-left panel is the reduced $\chi^{2}$ obtained from the stacked profile relative to the corresponding stacked median and variance at each time. Middle panel: spectrum vs. phase diagram for the best P0 and DM. Bottom-middle panel: reduced $\chi^{2}$ obtained for each trial DM. Bottom-right panel: reduced $\chi^{2}$ obtained for each trial (P0 and P0 time derivative, $\dot{P}$ ) at the best DM. Top-right panels: reduced $\chi^{2}$ relative to $\dot{P}$ for the best $\mathrm{P} 0$ and relative to $\mathrm{P} 0$ for the best $\dot{P}$.

Fig. 7, there is a frequency dependent structure in parameters $Q$ and $U$. This is the signature of an imperfectly corrected Faraday effect caused by ionospheric variations (i.e. the total RM is different from the value in the reference ephemeris used during the observation). Panel 9 shows the dynamic spectrum of the amplitude of the pulsation (recorded in the "ON-pulse" region), which can be used to quantify mitigation and data quality. Panel 10 is the dynamic spectrum of the bandpass (spectral response from the "OFF-pulse" regions) that can be used to assess the quality of the observation. The scintillation of the source is visible in panel 9, while panel 10 shows a 6 min periodic drop in the bandpass (gain) that results from the analog pointing of the instrument and can be corrected in post-processing.

\subsection{Data archive}

Currently, NenuFAR pulsar data are transferred to the Nançay Data Center (CDN) for temporary storage and distribution within the community. At the CDN, the data are stored on a RAID 6 storage cluster. Pulsar data recorded in folded mode (Sect. 3.4.1), single-pulse mode (Sect. 3.4.2), and dynamic spectrum mode (Sect. 3.4.4) will be kept at the $\mathrm{CDN}$, whereas waveform data (Sect. 3.4.3) and data recorded in UDP waveform mode (Sect. 3.4.5) are usually only kept temporarily and are removed after processing. A dedicated NenuFAR Data Center is currently being commissioned; it will have open access user interfaces to data products.

\section{Commissioning and first early science results}

The early science phase of the instrument will last until the end of 2021. Until then we will be conducting a set of observational programs with NenuFAR, under the umbrella of the Pulsars Key Science Project (ES03). For now, all our observations have been made with a limited version of the full instrument, that is, the 56 MAs first step configuration that started operating with a stable setup in February 2019 (Zarka et al. 2020). An 80 MAs configuration will become operational in the year 2021.

\subsection{Details of the emission profile}

The high sensitivity of NenuFAR and its stable gain across the band make it ideally suited for studying average profiles in detail. Figure 8 shows the evolution of the observed profile for the historical pulsar B1919+21, discovered by Jocelyn Bell in 1967 (Hewish et al. 1968). Using NenuFAR, this pulsar was detected with a high $\mathrm{S} / \mathrm{N}(>2000)$ down to $20 \mathrm{MHz}$ 
Single pulse results for 'search'

Source: B0531+21

Telescope: nenufar

Instrument: LUPP|

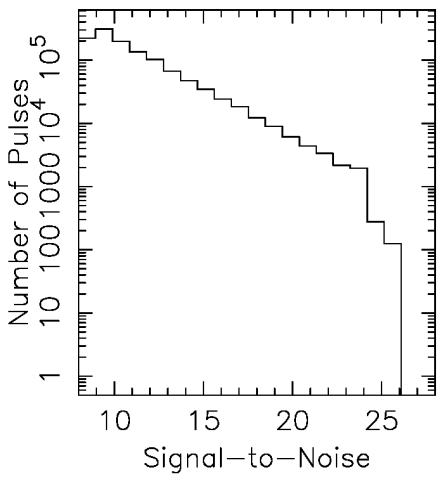

RA (J2000): $\quad$ 05:34:31.9738 DEC (J2000): 22:00:52.0632 $M_{\text {topo }}: 58910.751388889126$
N samples: 1956864

Sampling time: $2621.44 \mu \mathrm{s}$

Freq $_{\mathrm{ctr}}: 68.7 \mathrm{MHz}$
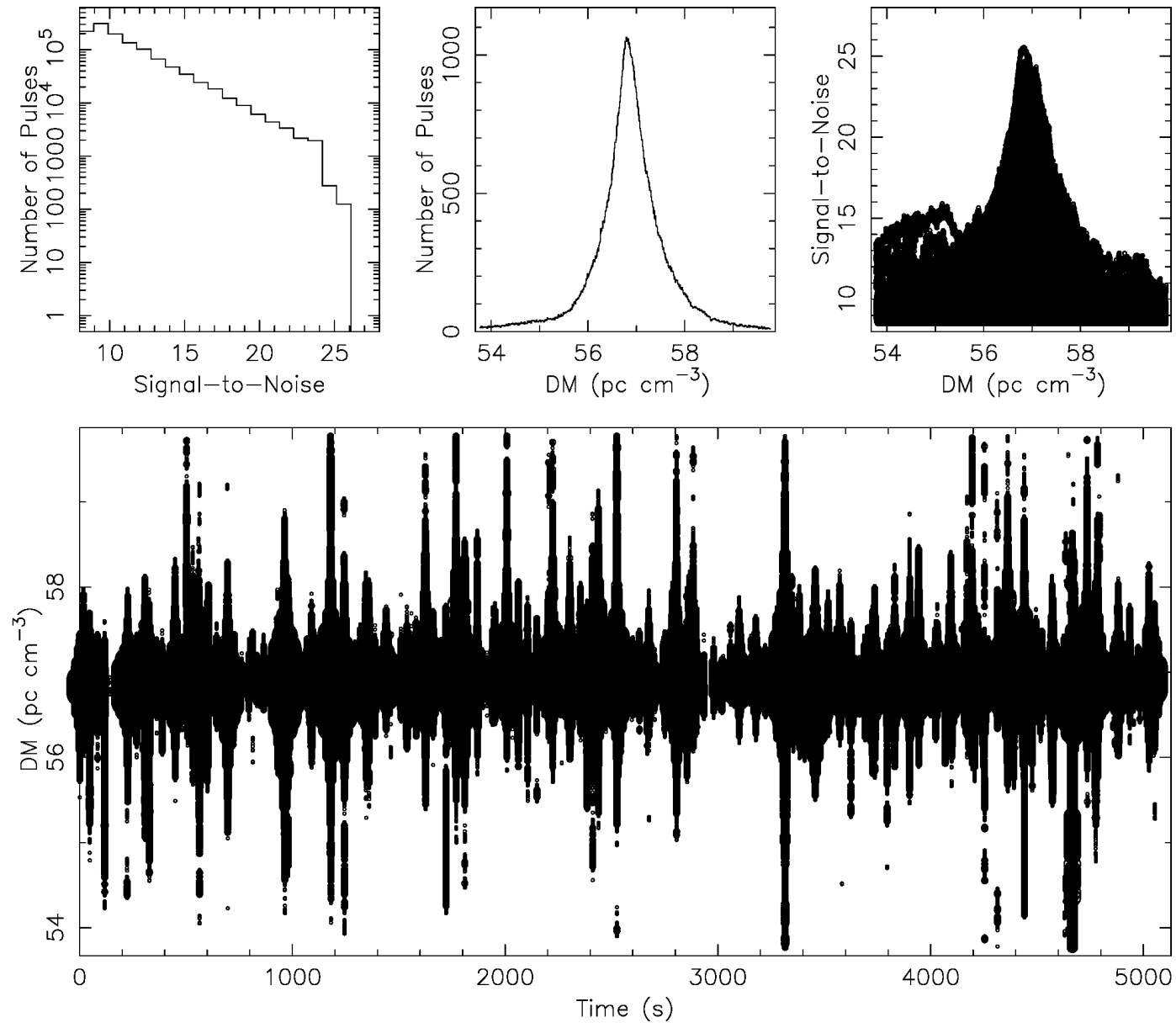

Fig. 6. Giant pulses from the Crab pulsar (PSR B0531+21) detected as a function of time and trial DM values. Bottom panel: detection time for all trial DMs. Due to the pulse width and scattering the same pulse can be detected over a wide range of DMs and count as multiple detections in the top panels. Top-left and top-middle panels: histograms of the S/Ns of GPs and of the trial DMs. Top-right panel: histogram of the S/Ns of GPs vs. trial DM values.

in only $22.5 \mathrm{~min}$. Obtaining good quality data at such low frequencies is important for modeling profile evolution and constraining emission heights. About three dozen sources have been observed with such frequency resolution, for example in integrations from 20 min to multiple hours as in Fig. 8 (Bondonneau 2019; Bondonneau et al. 2020).

\subsection{Crab pulsar giant pulses}

It has long been known that the Crab pulsar emits so-called giant pulses (GPs), very brief bursts of radio emission with intensities that exceed the intensity of an average pulse many times over (e.g. Howard et al. 1968; Cordes et al. 2004; Hankins et al. 2016; Rudnitskii et al. 2016). However, at such low frequencies these pulses are highly scattered (at $60 \mathrm{MHz}$, the scattering timescale is five times the pulsar's spin period) and consequently difficult to observe in folded mode. There have been a few singlepulse campaigns to observe GPs at low frequencies: For example, Popov et al. (2006) reported on the detection of 45 pulses in $12 \mathrm{~h}$ with the Ukrainian UTR-2 radio telescope below $30 \mathrm{MHz}$;
Eftekhari et al. (2016) announced the detection of 1400 pulses in $76 \mathrm{~h}$ of observation with the LWA1. With NenuFAR we detected 1000 pulses in only $1.4 \mathrm{~h}$ at a frequency of $68.7 \mathrm{MHz}$ (Fig. 6). Due to the amplitude of the scattering it is not possible to decide whether thez are anomalously intense individual pulses or actually GPs. However the statistics of events is comparable here to what is observed at higher frequencies and truly recognized as GPs. This detection rate and the telescope's sensitivity are very promising for future NenuFAR blind searches for new pulsars in the northern hemisphere. A strong individual pulse of this observation as a function of the observed frequency is shown in Fig. 9. At $52 \mathrm{MHz}$, the scattering tail of this pulse is roughly 20 times longer than the pulsar spin period.

\subsection{Drifting sub-pulses in PSR B0809+74}

Drifting patterns are a common phenomenon in pulsars (e.g. Weltevrede et al. 2006, 2008; Basu et al. 2019). Many of them show systematic variations of their substructures in an organized manner. Among the $\sim 70$ known pulsars that exhibit 

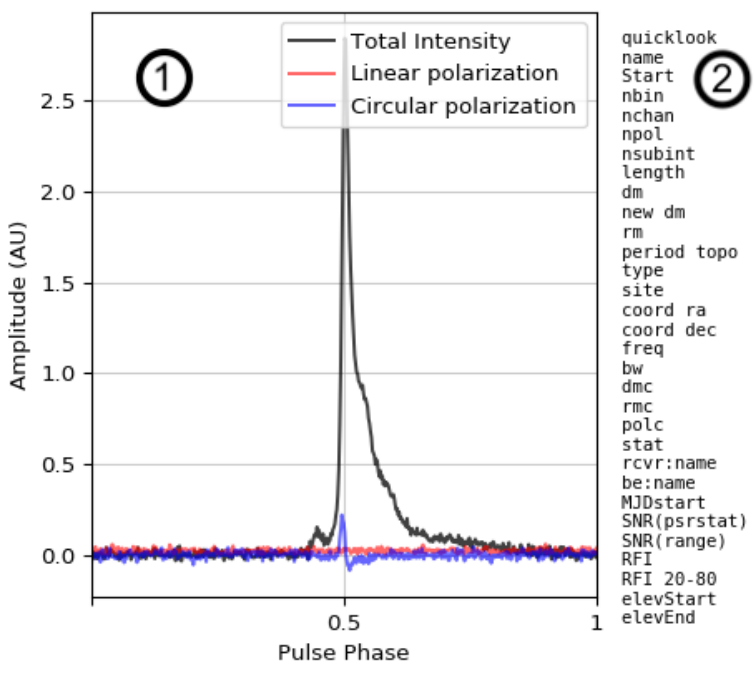

v01.00.00

Source name
2020-03-08 16:10:09.863 Number of pulse phase bins Number of polarizations Number of sub-integrations observation duration ( $\mathrm{s}$ ) $\begin{array}{ll} & \\ & 26.768000\end{array}$ \begin{tabular}{ll}
-63.7 \\
\hline
\end{tabular} Folding period (s) $\quad 0.714579680313$ Dbservation type Telescope name Source coordinates (hms) Source coordinates (dms) ( Dispersion corrected Faraday Rotation corrected Polarization calibrated Data state

Name of the backend instrument MJD of the first subintegration ignal noise ratio Signal noise ratio with snr.py Radi Frequency Interferency Elevation of the first subintegration 82.61
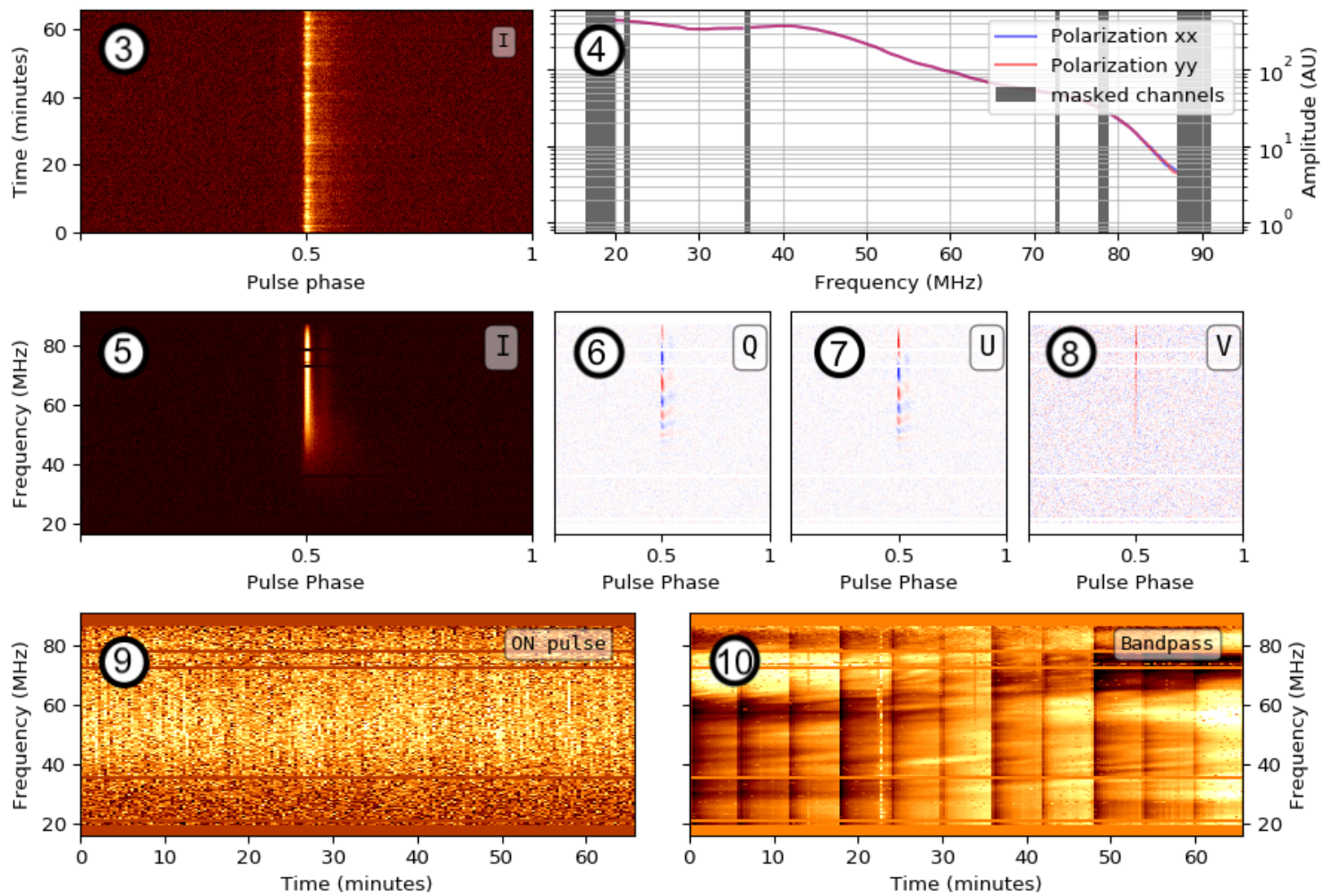

Fig. 7. Quicklook of an observation of PSR B0329+54 with NenuFAR in folded mode. Panel 1: profile of the pulsar in total intensity (black), linear polarization $\left(\sqrt{\left(Q^{2}+U^{2}\right)}\right.$; red), and circular polarization ( $V$; blue). Panel 2: metadata of the observation. Panel 3: time variation of the frequency-integrated profile. Panel 4: bandpass (spectral response of the telescope) for the linear feed polarization parameters XX and YY (in blue and red, respectively). Panels 5-8: frequency-resolved profiles for the Stokes parameters $I, Q, U$, and $V$. Panel 9: dynamic spectrum of the amplitude of the pulsation ("ON-pulse" region). Panel 10: dynamic spectrum of the bandpass (summed on "OFF-pulse" regions).

this behavior, an interesting prototype of this phenomenon is the bright and extensively studied pulsar B0809+74. It was observed down to $16 \mathrm{MHz}$ with LOFAR LBA (Stappers et al. 2011; Hassall et al. 2013) and detected in the 18-27 MHz band with UTR-2 (Zakharenko et al. 2013). The drifting substructures and the anomalously intensive pulses (AIPs) of PSR B0809+74 (AIPs, Ulyanov et al. 2006) are resolved in great detail by NenuFAR (Fig. 10) and are visible all along its frequency domain, from 85 down to $10 \mathrm{MHz}$. Because of the large fractional bandwidth and good sensitivity, NenuFAR data are very well suited for studying the frequency-dependent phase delay of drifting sub-pulses. This delay can provide novel constraints on the location of emission regions in the magnetosphere (Bilous et al. 2019; Maan 2019).

\subsection{Detection of $12 \mathrm{MSPS}$}

At frequencies below $100 \mathrm{MHz}$, only five millisecond pulsars (MSPs) had been detected prior to this work. The first detection of PSR J2145-0750 below $100 \mathrm{MHz}$ was published in Dowell et al. (2013), of PSRs J0030+0451 and J0034-0534 in Stovall et al. (2015), of PSR J1400-1431 in Swiggum et al. (2017), and, finally, of PSR J0437-4715 in Bhat et al. (2018). The first three of these MSPs were also detected with the LOFAR 


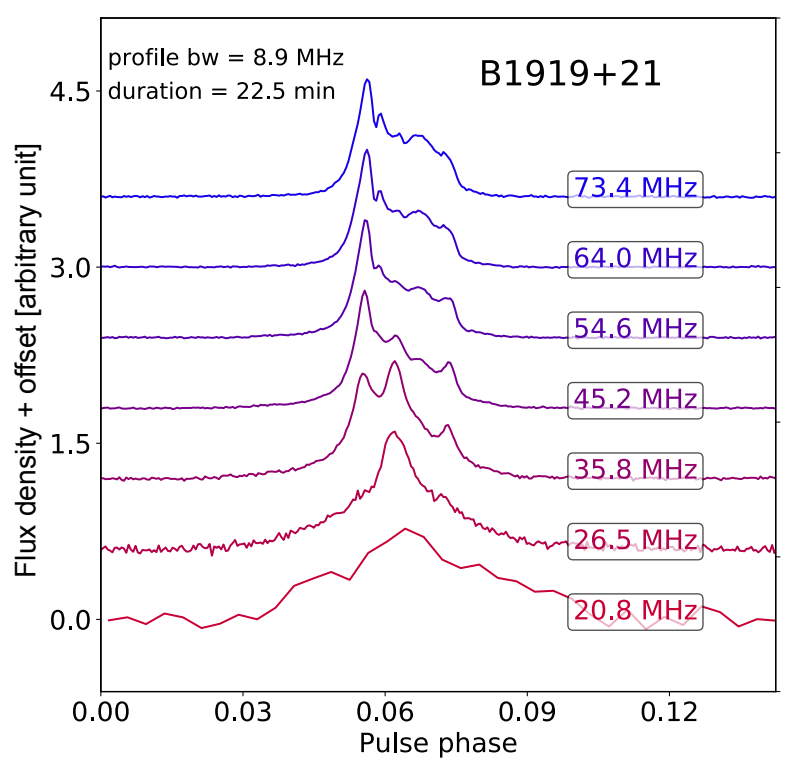

Fig. 8. Profile variation of PSR B1919+21 with frequency. Each line corresponds to an integrated and normalized profile on a frequency band of $8.9 \mathrm{MHz}$. The total integration time is $22.5 \mathrm{~min}$.

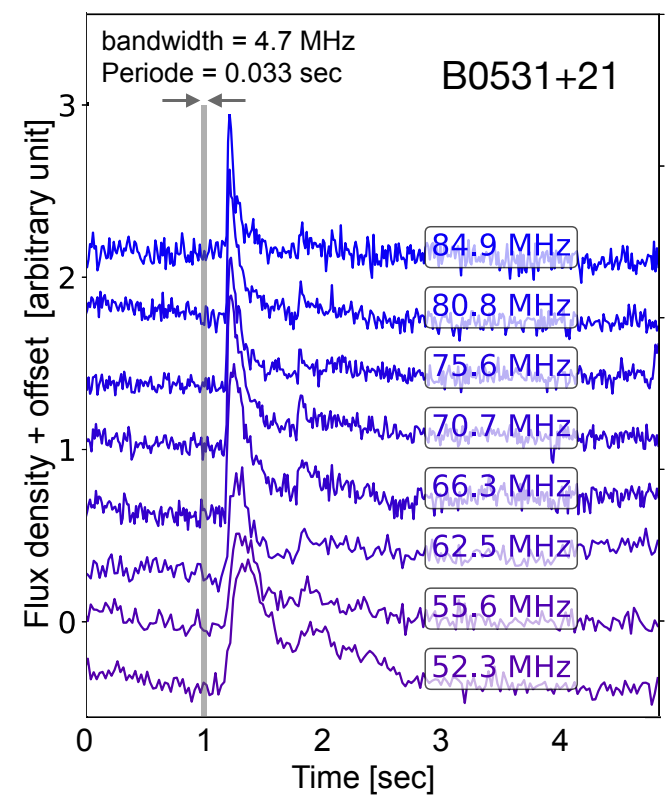

Fig. 9. Giant pulse from the Crab pulsar as a function of time and frequency. The gray area represents a single period of the pulsar.

LBA core by Kondratiev et al. (2016). Thanks to NenuFAR, we have already doubled this list. For each MSPs Table 2 lists the observing duration, the $\mathrm{S} / \mathrm{N}$, the DM and the mean flux density. As an example, in Fig. 11 we show the observed average pulse profile of PSR J1022+1001, obtained during the commissioning phase of the instrument. It was detected with a DM of $10.25356(3) \mathrm{pc} \mathrm{cm}^{-3}$, which is compatible with observations at higher frequencies, and a $\mathrm{S} / \mathrm{N}$ of 52 , corresponding to a mean flux density of $31 \pm 16 \mathrm{mJy}$ (following the same method that used to calculate the mean flux density limit in Sect. 2.1).

\subsection{DM variations from pulsar timing}

The study of time variations in DMs for different lines of sight enables studies of the ionized ISM and its turbulence (see, e.g.

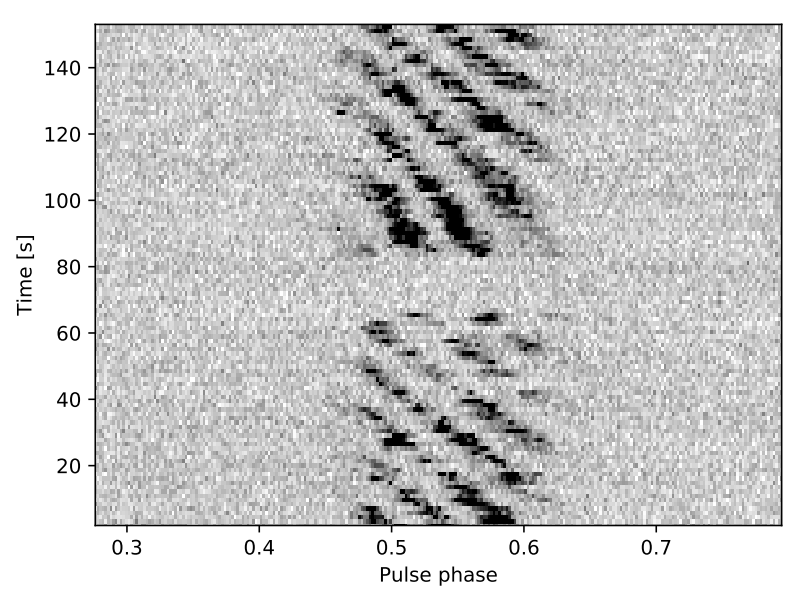

Fig. 10. Drifting sub-pulses of PSR B0809+74 in a 4 min observation over a bandwidth of $75 \mathrm{MHz}$ centered at $47 \mathrm{MHz}$. Each line represents a single-pulse period. A nulling phase of $\sim 20 \mathrm{~s}$ in duration is clearly visible one minute after the start of the observation.

Table 2. Millisecond pulsars detected by NenuFAR (eight of which are detected for the first time below $100 \mathrm{MHz}$ - they are marked with an asterisk).

\begin{tabular}{lcccc}
\hline \hline $\begin{array}{l}\text { Millisecond } \\
\text { pulsar }\end{array}$ & $\begin{array}{c}\text { Duration } \\
(\mathrm{min})\end{array}$ & $S / N$ & $\begin{array}{c}\mathrm{DM} \\
\left(\mathrm{pc} \mathrm{cm}^{-3}\right)\end{array}$ & $\begin{array}{c}\text { Mean flux } \\
(\mathrm{mJy})\end{array}$ \\
\hline J0030+0451 & 184 & 33 & $4.3331(1)$ & $67(34)$ \\
J0034-0534 & 178 & 46 & $13.76530(1)$ & $1168(584)$ \\
J0214+5222* & 178 & 7 & $22.0392(3)$ & $5(3)$ \\
J0740+6620* & 142 & 9 & $14.9620(1)$ & $6(3)$ \\
J1022+1001* & 178 & 52 & $10.25356(3)$ & $31(16)$ \\
J1231-1411* & 126 & 10 & $8.0918(1)$ & $34(17)$ \\
B1257+12* & 178 & 14 & $10.1539(1)$ & $25(13)$ \\
J1400-1431 & 120 & 98 & $4.93380(1)$ & $386(193)$ \\
J1658+3630* & 23 & 9 & $3.045(1)$ & $12(6)$ \\
J1710+49* & 178 & 7 & $7.0854(1)$ & $4(2)$ \\
J1806+2819* & 178 & 28 & $18.6732(1)$ & $30(15)$ \\
J2145-0750 & 178 & 50 & $9.0053(1)$ & $90(45)$ \\
\hline
\end{tabular}

Notes. For each of them the observing duration in minutes, the $\mathrm{S} / \mathrm{N}$ of the detection, the best-fit DM calculated using pdmp (part of the software package PSRCHIVE), and the mean flux density determined with an error bar of $50 \%$ from a bandwidth of $75 \mathrm{MHz}$ centered at $50 \mathrm{MHz}$ are listed.

Phillips \& Wolszczan 1991; Backer et al. 1993; Donner et al. 2019). This is particularly interesting at low frequencies, where the dispersive time delay is amplified by its dependence on $v^{-2}$. Such observations demand a large instantaneous fractional bandwidth, high sensitivity, and good clock stability, all of which are provided by NenuFAR.

Dispersion measure variations are typically studied via pulsar timing and the calculation of pulse times-of-arrival (TOAs). Initial tests have shown that for NenuFAR, the typical uncertainties of TOAs are 5-8 times smaller than for the LOFAR station FR606 (used in LBA mode, i.e. in the same frequency range). This translates to an increased precision for the study of DM variations. Figure 12 shows DM variations for PSR B1919+21, as measured by NenuFAR and LOFAR FR606 obtained with a 2D template fit (a phase-frequency template extending over 118 channels, built from a $10 \mathrm{~h}$ observation of PSR B1919+21 by NenuFAR, showing a $S / N>6000)$. The data from both 


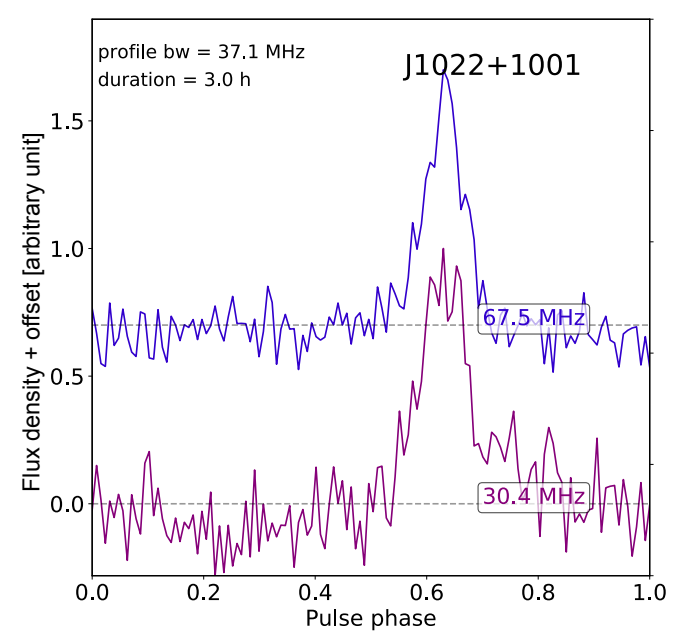

Fig. 11. First detection of the MSP J1022+1001 at frequencies below $100 \mathrm{MHz}$. The observation is integrated over $3 \mathrm{~h}$ and $37.1 \mathrm{MHz}$ of bandwidth at two central frequencies $(30.4$ and $67.5 \mathrm{MHz})$ in order to study the frequency dependence of the profile.

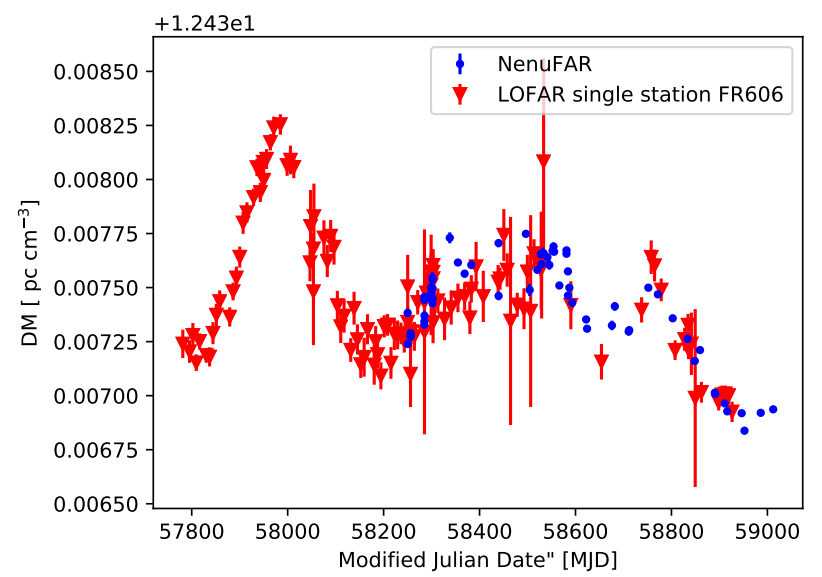

Fig. 12. Dispersion measure variations for PSR B1919+21 as measured by NenuFAR (blue dots) and the LOFAR single station FR606 (red triangle). For NenuFAR measurements, the error bar of the DM value is smaller than the dot size.

telescopes are comparable, but the NenuFAR data provide a much higher precision (on the order of $10^{-5} \mathrm{pc} \mathrm{cm}^{-3}$ ). As the template used for pulsar timing is derived from the data themselves, the procedure only gives relative DM values (sufficient for the analysis of DM variations) rather than absolute DM values (Donner et al. 2019). Series of DMs are well aligned especially if we look at the simultaneous observation in the left panel of Fig. 12.

\subsection{Interstellar scintillation studies}

By recording data in waveform mode, an additional filter bank step can be applied to increase the frequency resolution (similar to the dynamic spectrum recording mode) before phaseaveraging the observations (as in the folded mode). This hybrid combination of observing modes allows dynamic spectra to be constructed from the pulsed $\mathrm{S} / \mathrm{N}$ with much higher frequency resolution than that allowed by the standard folded mode, as well as with more sensitivity than allowed by the standard dynamic spectrum mode. This is particularly useful for the study of interstellar and inter-binary scintillation (Rickett 1990) since the size
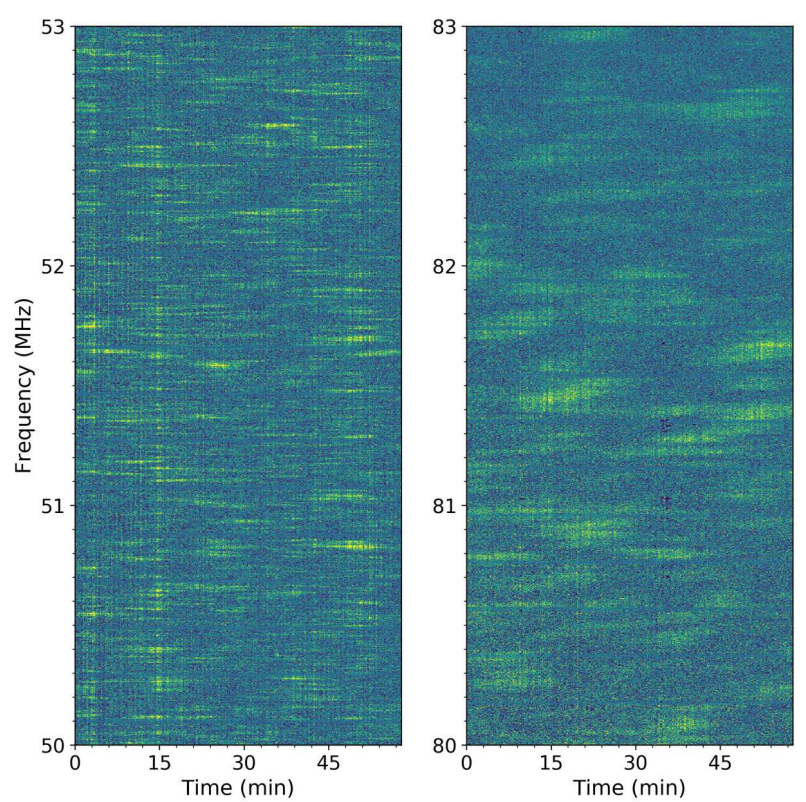

Fig. 13. Dynamic spectra of PSR B0809+74, in two $3 \mathrm{MHz}$ wide bands, of a one-hour NenuFAR observation. The color scale indicates the pulse $\mathrm{S} / \mathrm{N}$ ranging from blue (no signal) to yellow (high-S/N), which is heavily modulated due to diffraction in the ISM. The high-S/N "islands" are commonly referred to as scintles and provide information on the turbulent interstellar plasma.

of scintles is strongly dependent on the observing frequency, causing typical scintillation bandwidths to be much smaller than $195 \mathrm{kHz}$ at NenuFAR frequencies. Figure 13 shows an example of a dynamic spectrum that was obtained with this method on PSR B0809+74. The frequency resolution was set to $1.5 \mathrm{kHz}$, and the data were folded into $10 \mathrm{~s}$ integrations. The strong frequency scaling of the scintle size is apparent from the two $3 \mathrm{MHz}$ wide segments of data shown here: In the $50-53 \mathrm{MHz}$ range, the scintillation bandwidth (i.e., the size of scintles in frequency) is $3.9 \pm 0.7 \mathrm{kHz}$; in the $80-83 \mathrm{MHz}$ band, it is $23 \pm 1 \mathrm{kHz}$. The scintillation timescale (the size of the scintles in time) scales less strongly: from $3.79 \pm 0.07 \mathrm{~min}$ in the $50-53 \mathrm{MHz}$ subband to $6.1 \pm 0.3 \mathrm{~min}$ in the $80-83 \mathrm{MHz}$ band. This high sensitivity, and the fact that even a narrow frequency range can achieve numerous scintles, allows thorough and self-consistent statistical analyses of scintillation scaling laws and as well as precise monitoring of scintillation variations and their possible relation to other interstellar effects.

\subsection{Ongoing NenuFAR pulsar projects}

A first targeted survey has already allowed us to observe 650 pulsars with $\mathrm{DM}<100 \mathrm{pc} \mathrm{cm}^{-3}$ above $-20^{\circ}$ of declination, of which about $26 \%$ were detected (Bondonneau et al., in prep.). The nondetections are mainly due to the amplitude of the scattering at theses frequencies. These observations are an important complement to recent studies of the pulsar population observable below $85 \mathrm{MHz}$ such as Bilous et al. (2020) and Bondonneau et al. (2020).

This first NenuFAR catalog, combined with the first measurements of flux density and polarization, is now used as a basis for a set of follow-up programs, for example, the study of pulse-to-pulse variations with time and frequency, the effects of scintillation due to the ISM, the characterization of the pulsar population and the eclipses of certain systems. A set of 41 bright 
pulsars is tracked monthly to study the long-term variations of their average profiles, to characterize the DM evolution, and to study their spectral energy distribution in detail. Finally, a blind survey of the northern sky visible from Nançay started in July 2020 and will provide a more complete census of the local pulsar population at frequencies below $100 \mathrm{MHz}$.

\section{Summary}

We have provided a full description of the NenuFAR pulsar instrumentation, including the four main modes used for pulsar observations. We have shown a number of first results from our observations, part of which were obtained during the commissioning phase. These early science results are very promising and demonstrate the potential of NenuFAR for pulsar science; more detailed follow-up studies are currently being performed within the NenuFAR Pulsars Key Science Project. We expect significant contributions from NenuFAR, in particular in the fields of pulsar emission mechanisms and the study of the ISM and ionosphere.

With its high sensitivity at low frequencies, NenuFAR is also a milestone on the route to the Square Kilometer Array (SKA), hence its pathfinder label ${ }^{17}$. It will help refine the SKA science goals, particularly those concerning emission mechanism and propagation effects in the context of highly dispersed and scattered signals. As such, it will contribute to maintaining a useful bridge of pulsar studies over the coming decade and support the community of pulsar astronomers focusing on emission processes, populations, and ISM characterization. These science goals are complementary to those carried out at $L$ - and $S$-bands for high precision pulsar timing (gravitational wave detection and tests of general relativity in the context of the International Pulsar Timing Array [IPTA] Collaboration). When the SKA will becomes operational, joint studies between the SKA and NenuFAR will be performed in the two hemispheres in complementary frequency ranges, providing the potential for additional discoveries.

Acknowledgements. This paper is based on data obtained using the NenuFAR radio-telescope. The development of NenuFAR has been supported by personnel and funding from: Station de Radioastronomie de Nançay, CNRS-INSU, Observatoire de Paris-PSL, Université d'Orléans, Observatoire des Sciences de l'Univers en Région Centre, Région Centre-Val de Loire, DIM-ACAV an DIM-ACAV+ of Région Île-de-France, Agence Nationale de la Recherche. We acknowledge the use of the Nançay Data Center computing facility (CDN - Centre de Données de Nançay). The CDN is hosted by the Station de Radioastronomie de Nançay in partnership with Observatoire de Paris, Universite d'Orléans, OSUC and the CNRS. The CDN is supported by the Région CentreVal de Loire, département du Cher. This work was supported by the "Entretiens sur les pulsars" funded by Programme National High Energies (PNHE) of CNRS/INSU with INP and IN2P3, co-funded by CEA and CNES. The Nançay Radio Observatory is operated by the Paris Observatory, associated with the French Centre National de la Recherche Scientifique (CNRS).

\section{References}

Backer, D. C., Hama, S., Hook, S. V., \& Foster, R. S. 1993, ApJ, 404, 636 Basu, R., Mitra, D., Melikidze, G. I., \& Skrzypczak, A. 2019, MNRAS, 482, 3757

Bhat, N. D. R., Tremblay, S. E., Kirsten, F., et al. 2018, ApJS, 238, 1

Bilous, A., Kondratiev, V., Kramer, M., et al. 2016, A\&A, 591, A134

\footnotetext{
17 https://www.skatelescope.org/ precursors-pathfinders-design-studies/
}

Bilous, A. V., Watts, A. L., Harding, A. K., et al. 2019, ApJ, 887, L23 Bilous, A. V., Bondonneau, L., Kondratiev, V. I., et al. 2020, A\&A, 635, A75 Bondonneau, L. 2019, PhD Thesis, Université d'Orléans

Bondonneau, L., Theureau, G., \& Cognard, I. 2018, SF2A-2018: Proceedings of the Annual Meeting of the French Society of Astronomy and Astrophysics, 301

Bondonneau, L., Grießmeier, J. M., Theureau, G., et al. 2020, A\&A, 635, A76 Cameron, A. D., Champion, D. J., Bailes, M., et al. 2020, MNRAS, 493, 1063 Cordes, J. M., Bhat, N. D. R., Hankins, T. H., McLaughlin, M. A., \& Kern, J. 2004, ApJ, 612, 375

Demorest, P. 2014, Data Acquisition Software Primarily for the GUPPI Pulsar Backend, Github Repository

Desvignes, G. 2014, Coherent Dedispersion Software at Nancay: Clone of GUPPI by P. Demorest et al. - gdesvignes/NUPPI

Donner, J. Y., Verbiest, J. P. W., Tiburzi, C., et al. 2019, A\&A, 624, A22

Dowell, J., Ray, P. S., Taylor, G. B., et al. 2013, ApJ, 775, L28

DuPlain, R., Ransom, S., Demorest, P., et al. 2008, in Advanced Software and Control for Astronomy II, eds. A. Bridger, \& N. M. Radziwill, 70191D

Eftekhari, T., Stovall, K., Dowell, J., Schinzel, F. K., \& Taylor, G. B. 2016, ApJ, 829,62

Hankins, T. H., \& Rickett, B. J. 1975, Methods in Computational Physics. Volume 14 - Radio Astronomy (New York: Academic Press), 55

Hankins, T. H., Eilek, J. A., \& Jones, G. 2016, ApJ, 833, 47

Hassall, T. E., Stappers, B. W., Hessels, J. W. T., et al. 2012, A\&A, 543, A66

Hassall, T. E., Stappers, B. W., Weltevrede, P., et al. 2013, A\&A, 552, A61

Hessels, J. W. T., Ransom, S. M., Kaspi, V. M., et al. 2008, in 40 Years of Pulsars: Millisecond Pulsars, Magnetars and More, eds. C. Bassa, Z. Wang, A. Cumming, \& V. M. Kaspi, AIP Conf. Ser., 983, 613

Hewish, A., Bell, S. J., Pilkington, J. D. H., Scott, P. F., \& Collins, R. A. 1968, Nature, 217, 709

Hicks, B. C., Paravastu-Dalal, N., Stewart, K. P., et al. 2012, PASP, 124, 1090

Hotan, A. W., van Straten, W., \& Manchester, R. N. 2004, PASA, 21, 302

Howard, W. E., Staelin, D. H., \& Reifenstein, E. C. 1968, IAU Circ., 2110, 2

Keith, M. J., Jameson, A., van Straten, W., et al. 2010, MNRAS, 409, 619

Kondratiev, V. I., Verbiest, J. P. W., Hessels, J. W. T., et al. 2016, A\&A, 585, A 128

Lazarus, P., Brazier, A., Hessels, J. W. T., et al. 2015, ApJ, 812, 81

Lazarus, P., Karuppusamy, R., Graikou, E., et al. 2016, MNRAS, 458, 868

Lorimer, D., \& Kramer, M. 2004, Handbook of Pulsar Astronomy (Cambridge: Cambridge University Press)

Lorimer, D. R., Esposito, P., Manchester, R. N., et al. 2015, MNRAS, 450, 2185

Lynch, R. S. 2013, in Neutron Stars and Pulsars: Challenges and Opportunities after 80 Years, ed. J. van Leeuwen, IAU Symp., 291, 41

Maan, Y. 2019, ApJ, 870, 110

Malofeev, V. M. 1993, Astron. Lett., 19, 138

Malofeev, V. M., Malov, O. I., \& Shchegoleva, N. V. 2000, Astron. Rep., 44, 436

Manchester, R. N., Lyne, A. G., Camilo, F., et al. 2001, MNRAS, 328, 17

Manchester, R. N., Hobbs, G. B., Teoh, A., \& Hobbs, M. 2005, AJ, 129, 1993

Maron, O., Kijak, J., Kramer, M., \& Wielebinski, R. 2000, A\&AS, 147, 195

Parent, E., Kaspi, V. M., Ransom, S. M., et al. 2019, ApJ, 886, 148

Phillips, J. A., \& Wolszczan, A. 1991, ApJ, 382, L27

Pilia, M., Hessels, J. W. T., Stappers, B. W., et al. 2016, A\&A, 586, A92

Popov, M. V., Soglasnov, V. A., Kondrat'ev, V. I., et al. 2006, Astron. Rep., 50, 55

Ransom, S. M. 2001, PhD Thesis, Harvard University

Rickett, B. J. 1990, ARA\&A, 28, 561

Rudnitskii, A. G., Karuppusamy, R., Popov, M. V., \& Soglasnov, V. A. 2016, Astron. Rep., 60, 211

Sanidas, S., Cooper, S., Bassa, C. G., et al. 2019, A\&A, 626, A104

Sieber, W. 1973, A\&A, 28, 237

Stappers, B. W., Hessels, J. W. T., Alexov, A., et al. 2011, A\&A, 530, A80

Stovall, K., Ray, P. S., Blythe, J., et al. 2015, ApJ, 808, 156

Swiggum, J. K., Kaplan, D. L., McLaughlin, M. A., et al. 2017, ApJ, 847, 25

Ulyanov, O. M., Zakharenko, V. V., Konovalenko, O. O., et al. 2006, Radio Phys. Radio Astron., 11, 113

van Haarlem, M. P., Wise, M. W., Gunst, A. W., et al. 2013, A\&A, 556, A2

van Straten, W., \& Bailes, M. 2011, PASA, 28, 1

Weltevrede, P., Edwards, R. T., \& Stappers, B. W. 2006, Chin. J. Astron. Astrophys. Suppl., 6, 13

Weltevrede, P., Stappers, B., \& Edwards, R. T. 2008, in 40 Years of Pulsars: Millisecond Pulsars, Magnetars and More, eds. C. Bassa, Z. Wang, A. Cumming, \& V. M. Kaspi, AIP Conf. Ser., 983, 43

Zakharenko, V. V., Vasylieva, I. Y., Konovalenko, A. A., et al. 2013, MNRAS, 431, 3624

Zarka, P., Denis, L., Tagger, M., et al. 2020, URSI GASS 2020, Session J01 New Telescopes on the Frontier, https://tinyurl.com/ycocd5ly 